\title{
Impact of serum in cell culture media on in vitro lactate dehydrogenase (LDH) release determination
}

\author{
Bernhard Hiebl $^{\mathrm{a}, *}$, Sinem Peters ${ }^{\mathrm{b}}$, Ole Gemeinhardt ${ }^{\mathrm{c}}$, Stefan M. Niehues ${ }^{\mathrm{c}}$ \\ and Friedrich Jung ${ }^{\mathrm{d}}$ \\ ${ }^{a}$ Institute for Animal Hygiene, Animal Welfare and Farm Animal Behaviour, University \\ of Veterinary Medicine Hannover, Foundation, Hannover, Germany \\ ${ }^{\mathrm{b}}$ CREAMEDIX GmbH, Weingarten, Germany \\ ${ }^{\mathrm{c}}$ Department of Radiology, Charité-University Medicine Berlin, Berlin, Germany \\ ${ }^{\mathrm{d}}$ Institute for Clinical Haemostasis and Transfusion Medicine, University of Saarland, \\ Homburg/Saar, Gemany
}

\begin{abstract}
Cell exposure to cytotoxic substrates can cause decreased cell viability or cellular death through necrosis or apoptosis. The common characteristic of both reaction patterns (necrosis and apoptosis) is a compromised or damaged cell membrane, which results in a release of the cytoplasmatic enzyme lactate dehydrogenase (LDH) into the extracellular space. For this reason the content of this enzyme in the supernatants from cells can be used as an indicator of damaged cell membrane integrity and serve as a general means to assess cell viability by measuring plasma membrane permeability. However, cell culture media which are used to maintain and to support cell growth commonly need serum supplementation, and sera already contain various LDH amounts, which may increase background absorbance in LDH release assays. Additionally the serum content varies depending on the cellular needs. For this reasons we tested the effect of different serum loads (inactivated fetal bovine serum [FBS]; 1-10 vol\%) on the results of a commercially available LDH release assay (Cytotoxicity detection Kit, Roche) using 3T3 cells. An FBS concentration of 1 vol\% was found to still maintain 3T3 cell viability, and after cell exposure to a cytotoxic substrate (surfactant) the cellular LDH release was higher than in the cells which were grown with higher serum concentrations ( 5 and $10 \mathrm{vol} \%$ ). These results show that a serum content of $1 \mathrm{vol} \%$ is advantageous for cytotoxicity assays based on the LDH release, but due to the cellular differences in the need of serum components test results are limited to $3 \mathrm{~T} 3$ cells.
\end{abstract}

Keywords: Lactate dehydrogenase, biocompatibility, cytotoxicity, cell culture

\section{Introduction}

In test strategies to determine cytotoxic effects it is common to assess necrosis and cell death by quantifying plasma membrane permeabilization [1] based on the uptake or exclusion of vital dyes $[2,3]$, or on the release of radioactive isotopes, fluorescent dyes, or calcein-AM from prelabeled target cells [4-7]. There are also tests available which measure cytoplasmic enzymes released by damaged cells, where the amount of enzyme activity detected in the culture supernatant correlates with the proportion of lysed cells [8-11]. These enzyme release assays have been described

\footnotetext{
${ }^{*}$ Corresponding author: Bernhard Hiebl, Institute for Animal Hygiene, Animal Welfare and Farm Animal Behaviour, University of Veterinary Medicine Hannover, Foundation, Bischofsholer Damm 15, 30173 Hannover, Germany. Tel.: +49 511 856 8958; E-mail: bernhard.hiebl@ tiho-hannover.de.
} 
for alkaline and acid phosphatase, glutamate-oxalacetate transaminase, glutamate-pyruvate transaminase, and arginosuccinate lyase. However, their use has been limited by the low amount of these enzymes present in many cells, and by the elaborate kinetic assays required to quantitate most enzyme activities.

Korzeniewski and Callewaert described 1983 a highly sensitive cytotoxicity test which was able to quantify changes of the total cell number or the cell membrane integrity as a function of the amount of cytoplasmic lactate dehydrogenase (LDH) leakage into the medium [12]. LDH is a stable tetrameric enzyme, belonging to the 2-hydroxy acid oxidoreductase family, which catalyzes the simultaneous inter-conversion of pyruvate to lactate and nicotinamide adenine dinucleotide (NAD)H to NAD ${ }^{+}$. This reaction is commonly used by cell for anaerobic respiration.

If the cells are lysed prior LDH determination in the cell culture medium, a change in the total cell number results in a concomitant change in the amount of substrate converted. In this way the LDH release assay allows to measure the degree of inhibition of cell growth (cytotoxicity) caused by a test substrate. If cell-free aliquots of the medium from cultures given different treatments are assayed, then the extracellular amount of LDH activity can be used as an indicator of relative cell viability as well as a function of membrane integrity.

However, cells often necessitate serum supplementation of cell culture media, and serum contains a significant level of LDH activity which can be sufficient to significantly decrease the sensitivity of assay and/or obscure detection of cellular LDH completely. The study therefore aimed to assess the impact of different concentrations $(1,5$, and $10 \mathrm{vol} \%)$ of heat inactivated serum in a standard cell culture medium on the sensitivity of the LDH release assay.

\section{Materials and methods}

\subsection{Cells and cell culture}

As test organism a mouse embryo fibroblast cell line (NIH/3T3 cells, DSMZ) was used. A freshly prepared $3 \mathrm{~T} 3$ cell suspension $\left(10,000\right.$ cells $/ \mathrm{cm}^{2}$; passage number lower $\left.<25\right)$ was seeded on polystyrene based 96-multiwell plates and grown for $24 \pm 2 \mathrm{hrs}$ at $37 \pm 1{ }^{\circ} \mathrm{C}$ in humidified air $\left(5 \mathrm{vol} \% \mathrm{CO}_{2} / 95\right.$ vol $\%$ air) with Dulbecco's modified Eagle medium (DMEM w/o phenolred) supplemented with 1 vol\% of a penicillin $(10,000$ units $/ \mathrm{ml}) /$ streptomycin $(10,000 \mu \mathrm{g} / \mathrm{ml})$ mixture (Biozyme Scientific $\mathrm{GmbH})$ and 10 vol\% heat inactivated fetal bovine serum (FBS, Life Technologies $\mathrm{GmbH}$ ).

Cells were grown until a cell layer of $80 \%$ confluence was established. Thereafter, the initial cell culture medium was replaced by DMEM which was supplemented with 1, 5 or 10 vol\% FBS (FBS-1, FBS-5, FBS-10). DMEM additionally supplemented with 0.01 vol\% of a cytotoxic surfactant (Triton $\mathrm{X}-100$, Carl Roth $\mathrm{GmbH}$ ) was used as positive control (S-FBS-1, S-FBS-5, S-FBS-10).

After $48 \pm 2 \mathrm{hrs}$ of incubation in humidified air $\left(5 \mathrm{vol} \% \mathrm{CO}_{2} / 95 \mathrm{vol} \%\right.$ air $)$ at $37 \pm 1^{\circ} \mathrm{C}$ the lactate dehydrogenase (LDH) activity in the medium was analyzed.

\subsection{LDH assay}

LDH release was measured with a commercially available LDH assay kit (Cytotoxicity Detection Kit, Roche). Briefly, LDH which becomes released in the cell surrounding environment causes reduction of $\mathrm{NAD}^{+}$to NADH and $\mathrm{H}+$ through the oxidation of lactate to pyruvate. Thereafter, a catalyst (diaphorase) transfers $\mathrm{H} / \mathrm{H}^{+}$from $\mathrm{NADH}+\mathrm{H}^{+}$to a tetrazolium salt (iodonitrotetrazolium, INT) to form a red colored formazan salt. The amount of color produced is then colorimetrically measured $(n=8)$ at a wavelength of $492 \mathrm{~nm}$ by a spectrophotometer. 


\subsection{Statistics}

After background subtraction from each value the average absorption at $492 \mathrm{~nm}$ was calculated for each well $(n=8)$. Data are reported as mean value \pm standard deviation for continuous variables, and were analyzed by one-way ANOVA/Tukey analysis. A $p$ value of less than 0.05 was considered significant.

\section{Results}

The positive control confirmed the sensitivity and accuracy of the test system. After cell growth in the serum containing media for $48 \mathrm{hrs}$ (FBS-1, FBS-5, FBS-10) LDH activity was significantly lower than after cell growth in the media which were additionally supplemented with the cytotoxic surfactant (S-FBS-1, S-FBS-5, S-FBS-10, $p<0.05$ ). The increase of the LDH activity after cell exposure to the surfactant was linear dependent on the medium content of the serum, and the correlation between the LDH activity and the serum content of the medium was negative. In the surfactant containing medium supplemented with 10\% FBS (S-FBS-10) the LDH activity increased by $79.3 \%$, in the medium with $5 \%$ FBS (S-FBS-5) by $164.2 \%$ and in the medium with $1 \%$ FBS (S-FBS-1) by $314.3 \%(p<0.05$ each).

Without surfactant exposure to the cells the correlation between the LDH activity and the serum content of the medium was linear and characterised by a positive correlation. After cell growth for 48 hrs with FBS-10 the LDH activity in the medium was by $40.2 \%$ higher than after cell growth in FBS-1 and by $18.3 \%$ higher than after cell growth in FBS-5 $(p<0.05$, see Fig. 1).

\section{Discussion}

Determination of the cellular LDH release is a common method to assess functional integrity of the plasma membrane in cytotoxicity test portfolios. However, in vitro serum supplementation of the growth media is often necessary to maintain cell viability and proliferation, and the LDH activity of the supplemented serum can limit sensitivity of LDH release assays.

FBS is known to support the growth of many cell types by providing amino acids (e.g. histidine, isoleucine, leucine, lysine, methionine, phenylalanine, threonine, tryptophan, and valine), vitamins,

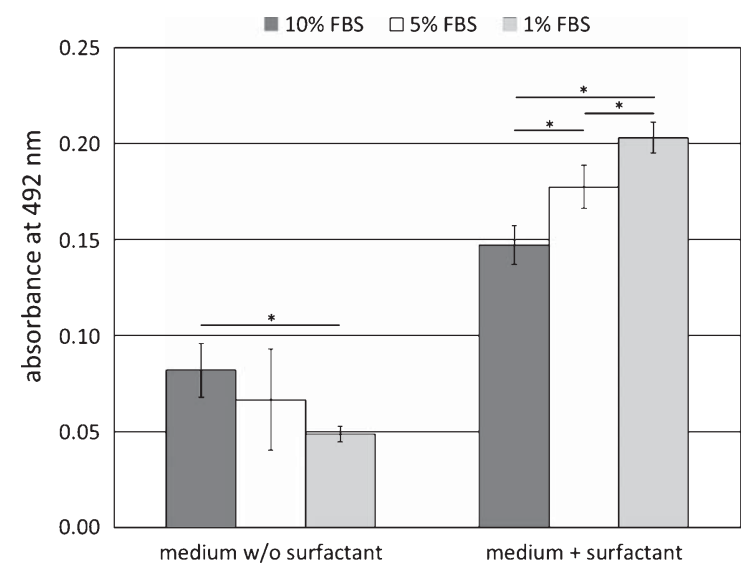

Fig. 1. LDH release after 3T3 growth in DMEM supplemented with 1, 5 or 10 vol\% fetal bovine serum (FBS); additional supplementation with 0.01 vol\% Triton X-100 was used as positive control; data represents mean \pm standard deviation. ${ }^{*} p<0.05$. 
inorganic minerals, fat, and nucleic acid derivatives required for cell culture [13]. For example, many vertebrate cells in culture need insulin for growth, and transferrin for iron incorporation including growth factors, attachment factors, iron transporters, vitamins, amino acids, lipids, carbohydrates, hormones, and trace elements.

The study showed a positive linear correlation between the serum concentration of cell culture medium and the LDH activity. Supplementation of the cell culture medium with a surfactant which served as exemplary cell toxin resulted in a significant increase of the extracellular LDH activity. This increase was seen in the cell culture media with high and low level serum supplementation and proved test sensitivity within the serum range of $1-10 \%$. The increase was also linearly dependent on the serum concentration of the medium, but the correlation between the serum content of the medium and the LDH activity was negative. This result can be explained by limited essential serum components in the media with the lower serum concentrations. The cytotoxic surfactant caused damages of the cell membrane, and the cellular mechanisms for cell membrane regeneration are dependent on sufficient supply of nutrients. The availability of these nutrients was higher in the medium which was supplemented with $10 \mathrm{vol} \%$ serum as in the medium with only 5 or 1 vol\% serum.

However, these results refer only to a cell culture medium which was supplemented with a surfactant. $\mathrm{LDH}$ release of cells which are exposed to cytotoxic radio contrast media for instance cause different results. Franke et al. have shown that the iso-osmolar contrast medium iodixanol and the low-osmolar contrast medium iopromide can interact with the LDH available in the culture medium as well as with the substrates delivered with the measurement system for the assessment of LDH activity, so that both, the amount of LDH and the activities of enzymes involved might be influenced [14].

In addition, it has to be taken into account that the optimal serum level in cell culture media depends on the target cell and their specific need, which might be much higher than for the 3T3 cells [15], resulting in higher cell independent LDH activities in the medium and reduced LDH assay sensitivity.

\section{References}

[1] Chan FK, Moriwaki K, De Rosa MJ. Detection of necrosis by release of lactate dehydrogenase activity. Methods Mol Biol 2013;979:65-70.

[2] Cook JA, Mitchell JB. Viability measurements in mammalian cell systems. Anal Biochem 1989;179(1):1-7.

[3] Jones KH, Senft JA. An improved method to determine cell viability by simultaneous staining with fluorescein diacetatepropidium iodide. J Histochem Cytochem 1985;33(1):77-9.

[4] Oldham RK, et al. Direct comparison of three isotopic release microtoxicity assays as measures of cell-mediated immunity to Gross virus-induced lymphomas in rats. J Natl Cancer Inst 1977;58(4):1061-7.

[5] Leibold W, Bridge S. 75Se-release: A short and long term assay system for cellular cytoxicity. Z Immunitatsforsch Immunobiol 1979;155(4):287-311.

[6] Danks AM, et al. Cellular alterations produced by the experimental increase in intracellular calcium and the nature of protective effects from pretreatment with nimodipine. Brain Res Mol Brain Res 1992;16(1-2):168-72.

[7] Kolber MA, et al. Measurement of cytotoxicity by target cell release and retention of the fluorescent dye biscarboxyethyl-carboxyfluorescein (BCECF). J Immunol Methods 1988;108(1-2):255-64.

[8] Decker T, Lohmann-Matthes ML. A quick and simple method for the quantitation of lactate dehydrogenase release in measurements of cellular cytotoxicity and tumor necrosis factor (TNF) activity. J Immunol Methods 1988;115(1): 61-9.

[9] Szekeres J, Pacsa AS, Pejtsik B. Measurement of lymphocyte cytotoxicity by assessing endogenous alkaline phosphatase activity of the target cells. J Immunol Methods 1981;40(2):151-4.

[10] Masanet J, Gómez-Lechón MJ, Castell JV. Hepatic toxicity of paraquat in primary cultures of rat hepatocytes. Toxicol In Vitro 1988;2(4):275-82. 
[11] Martin A, Clynes M. Acid phosphatase: Endpoint for in vitro toxicity tests. In Vitro Cell Dev Biol 1991;27A(3 Pt 1): 183-4.

[12] Korzeniewski C, Callewaert DM. An enzyme-release assay for natural cytotoxicity. J Immunol Methods 1983;64(3): $313-20$.

[13] Brunner D, et al. Serum-free cell culture: The serum-free media interactive online database. ALTEX 2010;27(1):53-62.

[14] Franke R, et al. Reduced diagnostic value of lactate dehydrogenase (LDH) in the presence of radiographic contrast media. Clinical Hemorheology and Microcirculation 2010;45(2-4):123-30.

[15] Barnes D, Sato G. Serum-free cell culture: A unifying approach. Cell 1980;22(3):649-55. 